\title{
Development of a points-based system for determining workload for a neonatology full-time equivalent
}

\author{
Steven Olsen $^{1} \cdot$ Howard Kilbride $^{1}$
}

Received: 4 June 2018 / Revised: 28 August 2018 / Accepted: 18 September 2018 / Published online: 5 October 2018

(c) Springer Nature America, Inc. 2018

\begin{abstract}
Quantifying the workload for a hospital-based physician can be a challenge. We developed a novel approach to providing equity and flexibility for physicians working in a large system with heterogeneous clinical activities. In developing this points-based system, expected clinical work hours were calculated for a full-time equivalent. A point value was assigned to each clinical service based upon the hours, complexity and intensity of the work. A hypothetical work schedule was created using the expected clinical hours and translated into points needed for a full-time clinician. Faculty appreciate the flexibility and feel appropriately valued and equitably paid. Our system serves as a recruitment tool and enables the division to better understand resource needs and predict the need for additional staff. Development of a point-based model for calculating clinical work has successfully created a homogeneous system to define and measure physician work expectations in a heterogeneous clinical environment.
\end{abstract}

\section{Introduction}

Physician clinical workload is often measured by number of clinics staffed, the number of patient contacts per day, or relative value units (RVUs) generated [1]. However, quantifying the workload for a hospital-based physician can be a challenge. Specifically, for a neonatologist, the workload depends on the size and scope of the neonatal intensive care unit (NICU) and expectations for night or weekend coverage. In addition, there are often other non-NICU clinical responsibilities as well as non-clinical work expectations.

For many neonatologists, clinical work has been defined by "weeks of attending service" each year. The number of weeks on service may vary based on academic or other nonclinical considerations. Neonatologists' clinical responsibilities are often unscheduled and cannot be postponed, so the clinical service must be planned for $24 \mathrm{~h}$ a day, 365 days a year. In the past, when the neonatologist was "on service," the expectation had been that he/she would be available to

Steven Olsen

slolsen@cmh.edu

1 Children's Mercy Kansas City, University of Missouri-Kansas City School of Medicine, Kansas City, MO, USA cover all clinical questions for the assigned weeks and would have no or limited clinical expectations at other times. In those scenarios, the "first-call" providers at night and weekends were in-house resident physicians. More recently, with changes in resident work hours, advanced practice nurses, or physician assistants have been available to assess and stabilize infants on off-hours. In these settings, neonatologists have provided supervision and planning on rounds during the daytime, but have generally only been available at night by phone. However, with increased complexity of neonatal care, concerns about safety, as well as marketing and billing realities, $24 \mathrm{~h}$ in-house neonatologist coverage has become the expectation [2]. In the new reality, it is no longer practical to consider clinical work as only "weeks on service".

In actuality, the hours spent by a neonatologist in daytime rounding represent a minority of clinical hours necessary to care for the NICU patients. For the current clinical neonatologist, the many hours of night, weekend, and holiday call in the NICU exceed the hours of daytime responsibilities. A more accurate way of quantifying clinical workload for a neonatologist is actual measurement of the work hours to accomplish the multiple clinical responsibilities. Hours to be counted would include those required for direct patient contact during weekday daytime, as well as hours spent in hospital during nights, holidays, and weekends; also included are hours for prenatal or floor 
consultations, delivery room attendance, outpatient clinic assignments, and other services mandated for effective care of patients.

Some units use RVUs to assess clinical workload, reflecting number and intensity of patients seen. However, although this approach may be reasonable in the ambulatory clinic setting, it is not an equitable measure in neonatology. The majority of revenue generated in the NICU is based upon $24 \mathrm{~h}$ billing codes. Although RVUs reflect effort for the entire $24 \mathrm{~h}$ period, the daytime neonatologist generally receives the credit and physicians working at night may receive less RVUs for their clinical contributions. In addition, neonatologists do not control admission patterns or directly affect the census of the NICU, patient acuity, or other factors that would modify RVUs. Thus, although neonatologists may spend equal hours on clinical work, work RVUs generated may show great variability between faculty members. It is not surprising that there is lack of correlation between work RVUs and clinical full-time equivalent (FTE) [3].

The main challenge for academic neonatology in this changing practice model is to determine how to distribute the workload equitably and still maintain enough flexibility in the work schedule. In an academic neonatal division, individuals may have different talents useful to meet clinical missions of the division. Assigning everyone the same distribution of clinical work doesn't provide the opportunity to individualize practice and match skills with the needed tasks. On the other hand, if each faculty member has different clinical responsibilities, the system needs to make work hours (and pay) equitable for all faculty. To address these complexities, our academic Division of Neonatology developed a scheduling and management system. This new quantitative system assigned points for each clinical activity, developing a model of "expected clinical points" that reflected the expected annual work hours per faculty member. The goal was to maximize efficiency of resources, while assuring that all staff had an equitable workload and met expectations for clinical work, as well as other professional requirements. The objective of this paper is to explain the development and initial outcomes of this novel approach to providing equity and flexibility for physicians working in a large system with heterogeneous clinical activities.

\section{Description of clinical service}

As part of the only free-standing children's hospital between St. Louis and Denver, Children's Mercy Hospital's Division of Neonatology, in the Department of Pediatrics, operates the region's only Level IV NICU. The 87-bed unit provides the acute and semi-acute care for moderate and critically ill newborns, including integrated subspecialty medical and surgical care for complex congenital conditions. In addition, the division provides neonatal management at seven community hospitals, varying in scope of service from small level II units to intensely busy level III units, which include coverage of more than 15,000 deliveries a year. The division is also responsible for the hospital's home ventilator program for former NICU patients who are discharged with tracheostomies, many still on mechanical ventilation. This high-risk infant follow-up provides care for infants and toddlers, offering daily clinics and 24/7 physician availability. Neonatologists also cover the Fetal Health Center, a perinatal program within the children's hospital offering prenatal care and delivery management for severe fetal conditions.

The division's leadership determined that clinical assignments include all aspects of clinical work, beyond weeks on service, to include night shifts, weekend on-call obligations, plus distinctions in duties derived from the variety of facilities: Level IV NICU, community Level III and Level II NICUs, the home ventilator program and other ambulatory services, and delivery backup and support at the Fetal Health Center. The division further determined that an efficient operation would take into account the talents, desires, and motivations of individual neonatologists on staff. The system must further account for changes over time as staffing and personal situations vary. The goal was the development of a homogeneous system for managing heterogeneous clinical activity with an equitable system of management.

\section{Creation of a value-based point system}

We calculated how many total work hours would be expected for each faculty member. We determined that a faculty member would spend approximately $50 \mathrm{~h}$ per week for 47 weeks per year for a total of $2350 \mathrm{~h}$ annually. The remaining 5 weeks included vacation and continuing medical education requirements. Based on the expectation that a clinician would spend $70 \%$ of professional time in direct clinical service, the system was developed for a clinical neonatologist to provide $\sim 1650$ clinical hours per year $(70 \%$ of $2350 \mathrm{~h}$ ). The remaining $\sim 700 \mathrm{~h}$ would be used for service to the division, hospital, and medical school, and would include administrative tasks, committee work, quality initiatives, and support for research.

The division director identified all responsibilities required to meet the clinical requirements and mission of the division. To begin, the number of hours needed for each clinical responsibility was determined. For example, at the level IV NICU, each assigned neonatologist was working on average in the hospital, $10 \mathrm{~h}$ per day. For each 2 -week 
Table 1 Points assigned for various daytime attending services

\begin{tabular}{|c|c|c|}
\hline Task & $\begin{array}{l}\text { Point value } \\
\text { per block* }\end{array}$ & $\begin{array}{l}\text { Point value } \\
\text { per day }\end{array}$ \\
\hline Level IV NICU & 6 & 0.6 \\
\hline \multicolumn{3}{|c|}{$\begin{array}{l}\text { Highest level of care, increased acuity, higher average hours to } \\
\text { complete tasks }\end{array}$} \\
\hline Level III (teaching) NICU & 5 & 0.5 \\
\hline \multicolumn{3}{|c|}{ May have lower census, but required teaching requirement } \\
\hline Level III NICU & 5 & 0.5 \\
\hline \multicolumn{3}{|c|}{ Patient load may be higher, but acuity lower } \\
\hline Community level II NICUs & 5 & 0.5 \\
\hline \multicolumn{3}{|c|}{ Lower census, lower acuity, may drive $>100$ miles/day for rounds } \\
\hline Home ventilator & 5 & 0.5 \\
\hline \multicolumn{3}{|c|}{$\begin{array}{l}\text { Inpatient consultations, busy outpatient clinic, } 24 / 7 \text { calls from home } \\
\text { nursing and parents }\end{array}$} \\
\hline High-risk fetal consult and delivery & 3 & 0.3 \\
\hline \multicolumn{3}{|c|}{$\begin{array}{l}\text { Prenatal consultations and attendance at high-risk deliveries, } \\
\text { average hours to complete are less than other services }\end{array}$} \\
\hline
\end{tabular}

Table 2 Points assigned for night and weekend calls, reflecting hours and intensity of work

\begin{tabular}{ll}
\hline Task & Point value \\
\hline System backup (including NICU, ECMO, Fetal Health & 0.1 \\
Center) & 0.2 \\
Home ventilator call weekday & 0.4 \\
Home ventilator call weekend & 0.5 \\
Level III NICU (teaching) weekend days & 0.8 \\
Community level II NICUs weekend days & 1.0 \\
Community NICUs weekday call night & 1.2 \\
Community NICUs weekend call night & 1.3 \\
Level III NICU weekend rounds daytime & 1.3 \\
Level IV NICU weekend rounds daytime & 1.4 \\
Level IV NICU (in-house) weekday call night & 1.7 \\
Level IV NICU (in-house) weekend call night &
\end{tabular}

block, then, each neonatologist attends approximately $100 \mathrm{~h}$ for the ten weekdays. The 2-week block does not include the weekends, which are counted separately. Although there was variability in daily workload at the level II NICUs, direct clinical work hours were, on average, $5 \mathrm{~h}$ per day or $50 \mathrm{~h}$ per 2 -week block. Next, each clinical activity was assigned a point value taking into account the hours needed to perform the work, as well as the relative complexity and intensity of the work. In assigning points for daytime attending weeks, the highest point value was given to the level 4 NICU rotation, recognizing the complexity of patient care, as well as the long hours. Other NICU settings required care for the same number or even more patients but with lower intensity, requiring fewer on-site hours, and little or no responsibility for trainee education, with variation of point values accordingly (Table 1). Similarly, point values for night and weekend coverage were assigned for each location and type of service. The points assigned ranged from the lowest, at 0.1 (for system backup or on-call for deliveries), to the highest, at 1.7 (for weekend nights at the Level IV NICU [Table 2]). The most difficult hours to estimate were those requiring backup call from home. We awarded a baseline value just for being on-call and added to this based on the estimated number of phone calls and average hours spent in the hospital providing patient care.

After assigning points for each clinical service, we calculated the number of points which reflected the expected hours of clinical work annually. To accomplish this "requirement", we created a hypothetical work schedule, which included attending weeks in the Level IV and Level III NICUs, night, weekend and holiday calls, which would total approximately $1645 \mathrm{~h}$ of clinical work. Based on that model, the standard requirement was determined to be 46 points for day attending (Table 1) and 80 points for night/weekend call (Table 2). In the hypothetical model, the number of points could be achieved with approximately 20 weeks of daytime attending, 40 night calls (20 in-house), 30 weekend day rounds, and a small amount of system backup call. However, depending on the actual services worked, an individual could attend on clinical services for more or less weeks and similarly, work more or less night calls to achieve the same point totals. Hypothetically, an individual physician could only work night calls and achieve enough points to equal the combined daytime and night/weekend call totals. The "nocturnist model" is not allowed in our academic division, as administrative meetings and educational lecture attendance are mandatory. However, it would be possible for a faculty member to do additional night calls with less daytime attending rotations. In addition, point requirements vary depending on non-clinical expectations. For physician scientists with extramural funding, the points needed to achieve are adjusted down based upon the grant funding requirement. Faculty with unique administrative or educational roles (division director, medical director, fellowship director) have a reduced point requirement (10-30\% depending on situation) to provide protected time from clinical responsibilities. In general, the point reduction is for daytime clinical responsibilities, with some specific situational modification for nighttime activity as needed.

The point system allows for flexibility and accounts for individual skills, desires, and service needs. Examples of flexibility and individual schedule variations include:

- Assuming more in-house night calls with fewer calls overall.

- Funded investigator incorporating weekend rounds and call with two subsequent night calls to complete a full 


\section{Comparisons of Call Frequency Based on Location}

Variation in Assignment Numbers Based on Work Location

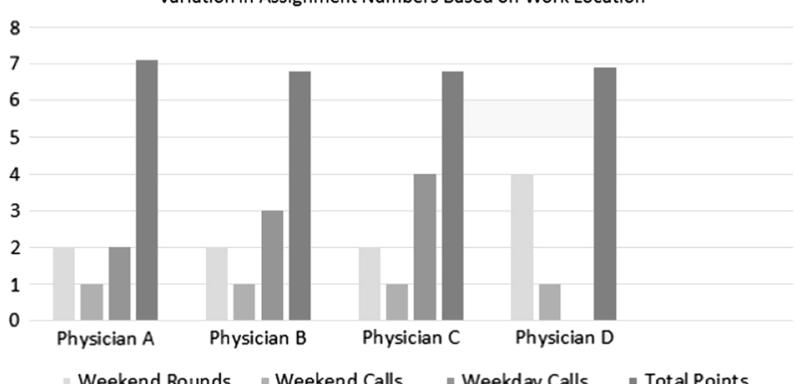

Fig. 1 Monthly assignment of weekend rounds and night call numbers for different physicians

month point requirement within 3-4 days to maximize time devoted to research.

- Senior clinical neonatologist doing more clinical service blocks and weekend rounds instead of night call.

Figure 1 depicts four different neonatologists' monthly weekend rounding and night call schedules. The total monthly points are nearly equal, whereas the number of calls and clinical work venues differ.

- Physician A has 2 weekend rounds at the Level IV NICU; 1 weekend Level IV in-house call; 2 weekday Level IV in-house call; about 7 points.

- Physician B has 2 weekend rounds in the busy community Level III NICU; 1 weekend community NICU call; 3 weekday calls at home for the Level II and III community NICUs; about 7 points.

- Physician $\mathrm{C}$ has 2 weekend rounds in the Level II and III community NICUs; 1 weekend call and 4 weekday calls at home for the Level II and III community NICUs; about 7 points.

- Physician D has 4 weekend rounds in the Level IV NICU; 1 weekend Level IV in-house call; no other calls; about 7 points.

Having a clear understanding of how many hours are expected for a clinical FTE is important for faculty members and department leadership. Faculty members deserve to have transparency in work expectations and compensation, as they seek work and lifestyle balance. At the same time, hospitals are under an increasing fiscal stress with decreases in third-party reimbursement. If the division director knows the expectation for work hours for a staff neonatologist and has determined the total number of clinical hours of work needed to cover the service, then determination of the number of clinical FTEs becomes a straightforward mathematical model, which can be succinctly presented to department leadership when requesting approval for additional staff.

\section{Validation and faculty response}

The proposed relative values for the clinical tasks were initially based primarily on average hours for each work responsibility. The values were discussed and vetted by the division members with some modifications made based on perceived differences in stress and effort in each setting. This model was then posed to an external neonatology division administrator with experience operating a division of relative size and complexity. The administrator did not have access to the proposed points but was provided descriptions of the clinical services and was asked to suggest relative values of one to the other. The relative point values returned strongly correlated with those proposed by the division for this model. With this validation, the system was launched for the division in 2007.

Faculty are given opportunity to request clinical rotations for each 6-month period. They may also indicate whether they would prefer only the "required points" or additional clinical responsibilities for additional points, if available. The clinical schedule of each neonatologist on staff is published and available for all using a computer program. At six-month intervals, point values are summarized by leadership and reviewed, with a goal of assuring that each neonatologist is on track to achieve the required number of points annually. To account for clinical hours above expectations, the model incorporated a payout based on the number of points over those required. The dollar value of a point was established to equate a night call in the NICU with the hospital's standard rate for overnight moonlighting.

From individuals' perspectives, staff neonatologists have reported they are satisfied that the work they do is appropriately valued and that they are equitably paid. They recognize their opportunities to achieve bonus payouts and appreciate the flexibility the system allows them in planning for time away, family needs, or other obligations. Each clinical staff member can track their own performance in points independently and has the flexibility to modify their personal targets to adjust to changing demands. As situations arise requiring a shift replacement (e.g., a staff member who becomes ill), faculty members are offered opportunities to assume the clinical responsibility and receive additional points that reflect these added efforts. Those substitutions occur seamlessly without concern, as staff appreciate that "extra" clinical work is being counted toward their point requirements, with decreased clinical requirements subsequently that year or a bonus payment.

During recruiting interviews, the potential new hires consistently report hearing positive views of the fairness of the work model and of the recognition that neonatologists 
receive through the additional points. Description of the model during recruitment has also satisfied applicants' requests to better understand specifics of work expectations. This feedback has shown us that the model serves as a valuable recruitment tool, particularly for providing transparency regarding expected clinical responsibilities and the flexibility allowed. The division administration has observed that the neonatal faculty welcome the opportunity to control their own time and appreciate that their clinical work is treated equitably.

Over the 10 years in which the model has been deployed, adjustments have been made to the point values. The quantification of the total clinical obligations of the division has created a clearer understanding of resource needs and better annual budgeting for the division. The model has been flexible as new clinical services and tasks have been added to the division responsibilities. Quantification of the diverse workload into points has created a clear guidance for predicting additional staffing needs. Thus, if providing services in a new NICU would require 138 attending points and 240 call night/weekend points, 3 new clinical FTEs would be needed (138 points/46 expected points per FTE and 240/80). The model has been validated and used to justify new clinical FTE requests for administration and hospital leadership. It appears that the point model can continue to be adapted for new complex services and to scale as the division grows.

\section{Comparison of the value-based point system to other clinical staffing models}

Determining work hour expectations is problematic for a clinical FTE in neonatology, for whom clinical work cannot be measured in standard "shifts" or regularly scheduled weekly work hours. In our system with varying clinical venues, involving differing average workloads, we found it especially challenging to establish equitable schedules for clinicians so that each felt the work they were doing was valued and recognized appropriately. We also noted that it is important to provide sufficient flexibility to keep services covered, while allowing staff to primarily work in areas where they perceived themselves to be most efficient and successful. To address these challenges, we have used a point-based model that quantifies the work effort for each clinical activity. In developing an annual point expectation for a clinician, we set a minimum expectation, with financial reward for additional clinical work productivity.

Our approach to recognizing and incentivizing clinical work for neonatologists varies from those of other practices, in which physician production uses the work component of the RVU. Several studies have used RVU benchmarks to construct performance-based compensation models and have succeeded in increasing productivity [4-6]. Other reports have reviewed incentive-based physician compensation programs, including RVUs as well as measures of academic productivity $[1,7]$. Compensation models in which varying percentages of salary is at risk based on productivity have resulted in increased supplemental pay $[8$, 9]. A neonatology group recently reported a revenue sharing plan, untethered to RVUs, in which multiple NICUs with different acuity were cooperatively staffed [10]. Using an approach similar to our point system, other medical groups have published models based on quantifying the workload time that allowed the group to objectively forecast staffing needs $[11,12]$. One could argue that neonatology or radiology practices, in which practitioners have little control over the number of patients or the billings, will not be incentivized appropriately with RVU measures and that models involving direct assessment of clinical hours are more realistic.

The flexibility allowed by the point-based system was also noted as a strength for those involved and specifically as a benefit for recruiting new faculty. Physician burnout, a work-related syndrome involving emotional exhaustion, depersonalization, and a sense of reduced personal accomplishment, has increased during the past decade and is dramatically higher than that of US workers in other fields $[13,14]$. The rate of burnout among physicians varies by clinical discipline, with many of the specialties at the front line—such as neonatology_-at highest risk. Literature cites various strategies that may reduce physician burnout, including effective leadership and schedule flexibility [13, 14]. Recognition of the need for greater schedule flexibility or protected time to pursue personally meaningful aspects of work (e.g., quality-improvement work, community outreach, research, education, or mentorship), may lessen risk of burnout.

The points-based system for our division of neonatology has proven to be a good model to address work-life balance issues. The system promotes fairness and equity among the staff, while giving individuals some control over work schedules and environments. Though we cannot prove a cause and effect relationship between the model and a reduced incidence of physician burnout, the inherent flexibility of the system supports continued job satisfaction in our division. Using this model, an individual physician can work a higher percentage of time in a level 4 NICU setting and decrease the number of overall weeks of clinical work; intermix the level 4 activity with level 2 and level 3 work to vary the time burden, patient acuity, and emotional burden of NICU care; or schedule clinical blocks in a shorter time interval to offer longer stretches of non-clinical time for academic pursuits, family or personal responsibilities, or any other priorities that could have an impact on personal development. 


\section{Limitations}

In making this model, several assumptions and limitations must be recognized. We assumed a $50 \mathrm{~h}$ work week and limited the number of weeks to 47 annually. Work hours for physicians vary by specialty and have been declining over the past decade. By self-report, physician work hours per week now average about $51 \mathrm{~h}$, similar to what is proposed in this model [15]. We also suggested that $30 \%$ of a clinical neonatologist's professional time be non-clinical. This percentage was based on expectations at our institution for mandatory in-servicing, responsibility for teaching medical students, residents, fellows and other trainees, participation in division and hospital-level quality-improvement initiatives, and other academic pursuits important for promotion. That percentage may be higher or lower at different medical centers depending on those expectations and departmentlevel input. As our system is designed for an academic neonatology division, adoption by a private practice group would require variations with different assumptions, including likely a higher percentage of clinical time to reflect differing group responsibilities. Alternatively, the percentage of clinical time could be increased to reflect the group's responsibilities. Further, our flexible model works well in our large division but may not be appropriate for a division with fewer neonatologists to share the workload. Similarly, a smaller division may not feel the up-front time commitment is worthwhile to gain consensus on how a points-based model would function. Additional study is needed to formally assess important outcomes, including improved physician efficiency and job satisfaction with the use of this type of system.

\section{Adaptation to other settings}

We propose that a points-based system could be helpful in determining physician workload for other inpatient clinical situations. Steps to develop a system would include:

- Make a list of all possible clinical work assignments/ responsibilities

- Determine the baseline clinical work responsibility (average assignment, shift, etc.) and award a value of 1

- Adjust every other clinical work responsibility either higher or lower from the baseline based upon hours onsite, perceived intensity (patient load and acuity), and life disruption (weekday vs. weekend, holiday, in-house vs. at home, etc.)

- Construct an overall clinical schedule that would meet the requirements of a full-time physician (weeks on service, number of calls, etc.). Use the schedule template to determine the total annual point requirement

\section{Conclusion}

The development of a point-based model for calculating clinical work has successfully created a homogeneous system to define and measure physician work expectations in a heterogeneous clinical environment. The model promotes manageable scheduling and provides an equitable workload for neonatologists, while meeting clinical service needs. This system appears to benefit both faculty members and leadership, yielding effective and efficient resource utilization.

Acknowledgements We wish to thank Penelope Bradley and Nancy Shawver for their assistance in preparation of this manuscript.

\section{Compliance with ethical standards}

Conflict of interest The authors declare that they have no conflict of interest.

\section{References}

1. Akl EA, Meerpohl J, Raad D, Piaggio G, Mattioni M, et al. Effects of assessing the productivity of faculty in academic medical centers: a systematic review. CMAJ. 2012;184:e602-e612.

2. Jobe AH, Martin RJ. 24/7 neonatal intensive care unit attending physician coverage: a clash of missions. Am J Respir Crit Care Med. 2010;182:729-31.

3. Gallagher E, Rapoza D. Issues and solutions in accurately measuring clinical full-time equivalents. J Pediatr. 2010;157:697-8.

4. Tarquino GT, Dittus R, Byrne D, Kaiser A, Neilson E. Effects of performance-based compensation and faculty track on the clinical activity, research portfolio, and teaching mission of a large academic department of medicine. Acad Med. 2003;78:690-701.

5. Andreae MC, Freed GL. Using a productivity-based physician compensation program at an academic health center: a case study. Acad Med. 2002;77:894-9.

6. Stites S, Steffen P, Turner S, Pingleton S. Aligning clinical compensation with clinical productivity: design and implementation of the financial value unit (FVU) system in an academic department of internal medicine. Acad Med. 2013;88:946-51.

7. Andreae MC, Blad K, Cabana M. Physician compensation programs in academic medical centers. Health Care Manag Rev. 2006;31:251-8.

8. Reich DL, Galati M, Krol M, Bodian CA, Kahn RA. A missionbased productivity compensation model for an academic anesthesiology department. Anesth Analg. 2008;107:1981-8.

9. Sakai T, Hudson M, Davis P, Williams J. Integration of academic and clinical performance-based faculty compensation plants: a system and its impact on na anesthesiology department. $\mathrm{Br} \mathrm{J}$ Anaesth. 2013;111:636-50.

10. LaGamma EF, Brumberg HL, Aboudi D, Shah S. Making a business case for a model of regionalization with neonatologist availability at all facilities: one institution's financial tell-all. J Perinatol 2018; e-pub ahead of print 6 July 2018; https://doi.org/ 10.1038/s41372-018-0157-0.

11. MacDonald SL, Cowan IA, Floyd RA, Graham R. Measuring and managing radiologist workload: a method for quantifying radiologist activities and calculating the full-time equivalents required to operate a service. J Med Imaging Radiat Oncol. 2013;57:551-7. 
12. Conlon M, Tharani Z. The implementation of a physician workload system in an academic health care setting: The Physician Activity Information System (PhAIS). BC Med J. 2008;50:565-70.

13. West C, Dyrbye L, Erwin P, Shanafelt T. Interventions to prevent and reduce physician burnout: a systematic review and metaanalysis. Lancet. 2016;388:2272-81.
14. Shanafelt T, Noseworthy J. Executive leadership and physician well-being: nine organizational strategies to promote engagement and reduce burnout. Mayo Clin Proc. 2017;92:129-46.

15. Staiger DO, Auerback DI, Buerhaus PI. Trends in the work hours of physicians in the United States. JAMA. 2010;303:747-53. 